\title{
SAÚDE E EDUCAÇÃO: CURSOS ALTERNATIVOS PARA DESENVOLVIMENTO DO PESSOAL DE ENFERMAGEM
}

\author{
Creusa Guimarães Madeira* \\ Silvia Angélica Jorge** \\ Seiko Kakehashi*** \\ Irma de Oliveira***
}

MADEIRA, C.G. et al. Saúde e Educação: cursos alternativos para desenvolvimento do Pessoal de Enfermagem. Rev.Esc.Enf.USP, v.30, n.2, p.217-28, ago. 1996.

Promover atividades educacionais terapêticas para o pessoal de enfermagem visando a diminuição do stress, a melhoria do relacionamento interpessoal e a busca do auto-conhecimento, são objetivos dos cursos promovidos pelo Departamento de Enfermagem da FCM e Serviço de Enfermagem em Educação Continuada do Hospital das Clínicas da UNICAMP. Através dos cursos: O Hospital e o Relacionamento Humano; Dança e Criatividade; Yoga e Relaxamento Mental, foram ministradas técnicas de respiração, relaxamento, sensibilização e conscientização corporal e de interpretação teatral. A estratégia empregada foi a "vivência grupal", com participação de atendentes, auxiliares e técnicos de enfermagem e enfermeiras, durante o horário de trabalho, em 15 a 20 encontros por curso. Como instrumento desta pesquisa foram utilizados os "depoimentos individuais", escritos pelos participantes, tendo como metodologia a análise de conteúdo. A avaliação demonstrou que a "Vivência" facilitou o relacionamento entre os membros da equipe de trabalho; abriu espaço para comunicação efetiva; favoreceu o auto-conhecimento; e ajudou na solução de problemas. Ficou evidente a importância da continuidade de cursos alternativos para auxiliar o funcionário na melhoria da relação consigo mesmo, com o outro, com o trabalho e valorizar a sua saúde/aprendizagem.

UNITERMOS: Educação em enfermagem.Relações interpessoais. Educação contínua.

\section{INTRODUÇÃO}

Pensar recursos humanos em enfermagem sob o ponto de vista da administração tradicional,é direcionar as ações do pessoal exclusivamente

\footnotetext{
* Enfermeira. Prof ${ }^{a}$ Assistente de Administração Aplicada a Enfermagem do Departamento de Enfermagem da FCM - UNICAMP.

** Enfermeira do Serviço de Enfermagem em Educação Continuada do Hospital de Clínicas - UNICAMP

*** Enfermeira. Prof ${ }^{a}$ Assistente da Área de Pediatria do Departamento de Enfermagem da FCM - UNICAMP
} 
para os interesses organizacionais, onde os indivíduos devem ser controlados e suas atividades dirigidas, se baseados nas teorias clássicas definidas por Taylor, Fayol e Weber.

As teorias humanísticas contribuiram nesta área, a partir da década de cinquenta para uma nova concepção de administração, que fundamentada nas ciências comportamentais, propicia o alcance dos objetivos individuais e organizacionais através de participação e compromisso do grupo. (TREVISAN ${ }^{10}$ )

Avançando um pouco mais, hoje a administração pode ser pensada dentro da abordagem holística, a qual preconiza a visão do ser humano na sua totalidade, se inter-relacionando e em interdependência com todos os fenômenos, nos aspectos físicos, psicológicos, biológicos, sociais e culturais. $\left(\mathrm{CAPRA}^{2}\right)$.

O holismo na administração surgiu a partir dos anos oitenta e vem sendo usado no Brasil, por algumas empresas subsidiárias e nacionais que empregam, entre outras, as idéias do ocultista russo Georgei Gurdjieff, para trazer as pessoas à consciência, derrubando conceitos de treinamento tradicional, chamando o pessoal para uma gerência mais participativa, e enfatizando o aspecto da mudança cultural da organização. (SEVO ${ }^{8}$ ).

Dentro desta proposta considera-se o homem como constituído de corpo, mente e espírito em harmonia e integração com o meio. Seu organismo é um sistema natural de cura sendo ele o responsável pelo desenvolvimento e manutenção do seu proprio bem estar. "O auto-conhecimento e a autorealização são importantes para o bem estar e a saúde e as crises e as doenças podem significar oportunidades para o crescimento ou evolução espiritual. A saúde holística tem abordagem multidisciplinar e utiliza sistemas alternativos para promoção, proteção e recuperação da saúde". (NOGUEIRA 7).

Acresce-se a isso que a prática da Administração não pode ser desvinculáda das ações educativas que são instrumentos que possibilitam o aumento do potencial do indivíduo ou do grupo contribuindo para sua autonomia, onde o mesmo transforma e é transformado através da prática crítica-criativa. (SILVA ${ }^{9}$ ).

Dentro desse processo educativo, não se pode ver o trabalhador apenas com necessidades de ser treinado para o trabalho, quando apresenta deficiências técnicas ou como agente de produção para dar mais lucro. Suas necessidades são mais abrangentes exigindo ser pensado de forma global: corpo, mente e espírito, participando e interagindo no seu ambiente de trabalho, se inter-relacionando com a natureza, e as pessoas, e em constante busca pelo seu crescimento.

Portanto, visando a integração do ser humano à dinâmica das relações sociais, na busca da realização de objetivos comuns à coletividade, adotou-se neste trabalho o processo de aprendizagem participativo no desenvolvimento 
das atividades (KOLLER ${ }^{3}$ ), dos cursos alternativos para desenvolvimento relativos à saúde e educação do pessoal de enfermagem, dentro do processo de educação institucionalizada, a nível hospitalar.

O intento da Administração de Pessoal de Enfermagem nesta instituição foi proporcionar a busca por condições de saúde e bem estar para os funcionários dentro da perspectiva holística e não apenas na visão cartesiana, onde o indivíduo é considerado de forma fragmentada e mecanicista.

A justificativa para esse procedimento surgiu de um trabalho de Integração Docente Assistencial do Departamento de Enfermagem da Faculdade de Ciências Médicas (FCM) com as unidades de Emergência Clínica e Cirurgia do Trauma do Hospital de Clínicas da UNICAMP. Através de um "levantamento de opinião" dos enfermeiros dessas unidades sobre suas funções, no qual foram detectados problemas de insatisfação e desmotivação, como pode-se observar por algumas das "opiniões" dadas por eles: - "Me sinto como trabalhador braçal"; - "Trabalho sem poder pensar, questionar e delegar.."; - "... estressada, desestimulada..."; - “... no final do plantão é como se nada tivesse feito". (MADEIRA ${ }^{5}$ ). Preocupados em buscar soluções para os problemas citados entre outras providências, foram consultados profissionais de diferentes áreas desta Universidade, que ap6s discussões sobre a complexidade das questões levantadas pelos enfermeiros, programou o curso: "O Hospital e o Relacionamento Humano", para os funcionários dessas duas unidades, onde se prestam cuidados a pacientes intensivos e semiintensivos.

Por solicitação e sugestão dos participantes desses cursos foram programados os demais: "Hata-Yoga"; "Dança e Criatividade"; "Relaxamento Mental: Religação com o Espiritual", abertos a todos os funcionários do hospital.

Todos eles tiveram como objetivos: a promoção de atividades educacionais terapêuticas visando a diminuição do stress; a melhora do relacionamento interpessoal e a busca do auto-conhecimento.

\section{DESENVOLVIMENTO DOS CURSOS}

Os cursos foram desenvolvidos no Hospital de Clínicas da Universidade Estadual de Campinas - HC - UNICAMP, em Campinas, São Paulo, que presta assistência de saúde à nível primário, secundário e terciário. No seu corpo de Enfermagem constam:- atendentes (234), auxiliares (478), técnicos de enfermagem (89) e enfermeiros (319), perfazendo um total de 1156 funcionários da categoria de Enfermagem, que trabalham em diferentes turnos: manhã, tarde e noite.

Frente aos problemas constatados na prática profissional diária e baseados na "observação" e nos "depoimentos" do pessoal de enfermagem,

Rev.Esc.Enf.USP, v.30, n.2, p.217-28, ago. 1996. 
estes cursos foram organizados e coordenados por uma docente do Curso de Graduação em Enfermagem da Faculdade de Ciências Médicas/FCMUNICAMP, com apoio do Serviço de Enfermagem em Educação Continuada do HC-UNICAMP.

Através do "intercâmbio multidisciplinar" foram contactados professores das áreas da Filosofia, Psicologia Educacional, Educação Física, Dança, Teatro, Recreação, Bioenergia, Yoga, ... pertencentes a diferentes Unidades desta Universidade e também profissionais de outras instituições. Nos contatos foram apresentados e explicados os objetivos dos cursos e o caráter de praticidade, utilidade e aplicabilidade que estes deveriam ter para o dia-adia das pessoas.

Na tentativa de atender certos aspectos peculiares a trabalhadores do hospital, foram considerados alguns critérios de tal forma que servissem para estimular e favorecer a participação do pessoal da enfermagem, como se segue:

O "horário" estabelecido obedeceu ao turno de trabalho do funcionário, para que este não dispendesse mais do seu tempo dentro da Instituição.

A “carga horária" foi prevista para que não sobrecarregasse aqueles que continuavam prestando assistência direta ao paciente na unidade. Foram utilizadas de 2 a 4 horas semanais num total máximo de 20 horas para cada curso, de forma que não sendo muito longo, pudesse atender maior número de funcionários, exceto o curso de yoga, que foi dado durante o ano todo, uma vez por semana.

O "local" foi uma sala de aula situada fora da unidade de trabalho, mas dentro do hospital, que apresentava espaço, luz natural, pouco ruído e condições mínimas para desenvolvimento das atividades.

Foram também providenciados colchonetes para os exercícios de relaxamento e realização de diversas técnicas específicas, bem como recursos audio-visuais, folhetos com o programa, transporte para alguns professores e lanche para facilitar a interação grupal.

Os cursos, a princípio se destinavam somente aos funcionários da equipe de enfermagem (enfermeiros, técnicos, auxiliares e atendentes de enfermagem) sem distinção de cargos, função, local de trabalho, sexo e idade. Pelo interesse demonstrado por outras pessoas, as vagas foram ampliadas.

Os "professores" que ministraram o curso foram escolhidos de acordo com a área de atuação e experiência de trabalho em grupos, e que apresentassem características de acessibilidade, fácil comunicação e com capacidade de desenvolver atividades práticas com grupos heterogêneos para atender às expectativas dos cursos. 


\subsection{CONTEÚDOS DOS CURSOS}

Foram temas do "O Hospital e o Relacionamento Humano" : Relação Transpessoal no hospital; Convivência Humana: o indivíduo e o grupo; Atitude profissional diante do paciente hospitalizado ou não; Conscientização corporal; A Coragem de brincar; Viagem Mental: instrumento para tomada de decisão; Improvisação de Sons com Máscaras; Corpo Moda ou Corpo Consciente; Antiginástica; Noções sobre Radiestesia e Radiônica; Dança e Arte: uma contribuição na formação do indivíduo.

"Danca e Criatividade" : Audio-tapes com estilos musicais diferentes; Cantigas e Samba de Roda; Provérbios; Pequenas estórias; Imitações de pessoas, animais e outros elementos da natureza.

"Hata-Yoga": Posturas (ASANA); Respiração (PRAMAYANA); Etica (YAMAMYAMA); Concentração (PRATIACHARA); Meditação (DYA NA); Meditação Transcendental (SANADHY).

"Relaxamento Mental: Religacão com o espiritual": Despertando a l;inguagem do coração; Técnicas para abrir os portões da criatividade; Visualização criativa; Afirmações; Meditação; A linguagem simbólica do dia-a-dia.

\subsection{ESTRATÉGIA UTILIZADA}

A escolha da estratégia "Vivência Grupal" ocorreu após a análise das condições do trabalho da população alvo, que atua diretamente na assistência ao paciente, vivenciando ocasiões de tensão constante que dificultam a percep̧̧ão de riscos para a sua saúde. Riscos estes, relacionados à complexidade da inter-relação funcionário-paciente; equipe de enfermagem e de saúde; trabalho rotineiro com ações mecanizadas; emprego de procedimentos e uso de equipamentos que nem sempre atendem aos princípios ergonômicos; e turno de trabalho que pode alterar alguns ritmos biológicos do funcionário.

A experiência de "vivência em grupo" foi realizada dentro de cada modalidade de atividades, de forma individual, em pares, em pequenos grupos e até envolvendo o grupo todo.

A dinâmica dos trabalhos permitia o envolvimento e a expressão dos participantes através do diálogo, depoimentos, histórias de vida, expressão corporal, conversas conjuntas e informais e relaxamento físico e mental, sendo dada ênfase nas técnicas:

- "Sensibilização e conscientização corporal: através do emprego de exercícios

que explorem o movimento das partes isoladas do corpo e a respiração";

- "Orientação espacial: direção, ritmo, ações básicas do esforço"; 
- "Expressão do movimento: criatividade, interpretação, dramatização";

- "Exercícios de hata-yoga, de anti-ginástica e recreação".

As atividades de um modo geral buscavam conduzir os participantes à interação interpessoal, à conscientização corporal, através dos movimentos, do relaxamento e auto-conhecimento, como pode ser verificado através de "depoimentos" de alguns participantes, descritos mais adiante.

\section{METODOLOGIA}

No presente estudo foi empregada a abordagem do método de análise do conteúdo que segundo BARDIN" "procura conhecer aquilo que está por trás das palavras sobre as quais se debruça..., numa busca de outras realidades através das mensagens". MINAYO ${ }^{5}$ acrescenta que este método enquanto "técnica de tratamento de dados, busca sua lógica na interpretação cifrada do material de caráter qualitativo". LUDKE ${ }^{4}$ explica que no "processo de decodificação das mensagens utiliza-se, não só o conhecimento formal, lógico, mas também o experencial, onde estão envolvidas sensações, percepções, impressões e intuições".

A população constituiu-se de 180 (cento e oitenta) funcionários, em sua maioria pertencentes a enfermagem e oriundos de diversas unidades de Internação, Serviços Especializados e Ambulatórios do HC-UNICAMP. Participaram também alguns escriturários, secretários, alunos de graduação em Enfermagem, funcionários do Colégio Técnico de Enfermagem, da Geociências e da Biologia, desta Universidade.

$\mathrm{Na}$ coleta de dados foram utilizados, como instrumentos, os "depoimentos individuais" escritos por 80 (oitenta) dos participantes, em sua maioria, atendentes, auxiliares e técnicos de enfermagem e enfermeiros. Eles opinaram de forma livre e aberta, escrevendo com suas proprias palavras sobre a experiência vivida nos cursos alternativos.

No tratamento dos dados, dentre as técnicas desta abordagem, utilizouse a análise temática, onde buscou-se encontrar os "núcleos de sentidos" que surgiram com maior freqüência nas falas escritas pelos participantes. Foram feitas repetidas leituras do material, determinando as palavras e frases-chave, as quais foram categorizadas e quantificadas. Para destacar os dados mais relevantes os resultados obtidos foram apresentados em números absolutos e índices percentuais, seguidos da busca de "significado das falas" e "sugestões" dadas pela população pesquisada.

As categorias de significados encontradas neste estudo foram: relacionamento humano: que foi subdividido em relacionamento inter-pessoal consigo mesmo/auto-conhecimento, e trabalho/instituição; aprendizagem; 
saúde física e mental; aplicabilidade na prática; reflexão; solução de problemas e pontos negativos, como podem ser observados na tabela 1.

Além disso, foram classificadas e quantificadas as sugestões dos participantes, apresentadas na Tabela 2.

\section{RESULTADOS E DISCUSSÃo}

Segundo os depoimentos dos participantes, o relacionamento interpessoal (22,0\%); aprendizagem $(20,2 \%)$; saúde física e mental $(18,5 \%)$ e relacionamento consigo mesmo/auto-conhecimento $(13,0 \%)$ foram categorias de possíveis parâmetros futuros, com significado relevante nos cursos alternativos, perfazendo mais de $73,0 \%$ do total, como mostra a Tabela 1.

TABELA 1 - Classificação de categorias de significados dos cursos alternativos para os participantes, segundo depoimentos individuais.Ano 1992.

\begin{tabular}{lll}
\hline CATEGORIAS DE SIGNIFICADO & $\mathrm{N}^{\circ}$ & $\%$
\end{tabular}

RELACIONAMENTO HUMANO: $\%$

- Interpessoal

- Consigo mesmo / Auto-conhecimento

- Trabalho / Instituição

Aprendizagem

Saúde Física / Mental

Aplicabilidade na Prática

Reflexão

Solução de Problemas

25

15

6

23

21

9

8

6

1

Pontos Negativos

TOTAL DE RESPOSTAS

Fonte: Cursos alternativos, Depto. Enf. FCM e SEEC - HC-UNICAMP.
22,0

13,0

5,2

20,2

18,5

8,0

7,0

5,2

0,9

Em relação a categoria de significado, "Relacionamento Humano" as falas dizem respeito a auto-conhecimento, relacionamento interpessoal e relacionam ento com trabalho/instituição. Estas, foram assim manifestadas:

\section{RELACIONAMENTO CONSIGO MESMO / AUTO-CONHECIMENTO}

- “.. consegui me conhecer melhor”, - “.. válido para observar melhor minha estrutura física e mental...”, - “... descoberta de uma pessoa que eu nõo conhecia que estava dentro de mim", - “... trabalhar a vida mais com emosões”, - “... nos faz voltar a visão para nosso eu", - "... senti-me um pouco gente". 


\section{RELACIONAMENTO INTERPESSOAL}

- "... foi a primeira abertura para amar a nós mesmos e juntos podermos trabalhar em favor dos nossos pacientes", - "... pude conversar, fofocar e conhecer colegas novas", - “... relacionamento com outras pessoas diferentes, convivência melhor com nossos supervisores, enfermeiras e atendentes", - "... proporcionou-nos um relacionamento maior entre pessoas funcionários e professores da UNICAMP”.

\section{RELACIONAMENTO COM TRABALHO / INSTITUIÇÃO}

- “.. serviu para eu entender melhor o meu ambiente de trabalho com todos os seus problemas", - “... uma ótima visão sobre como relacionar no meu ambiente de trabalho", - “... unindo setores e em matéria de hierarquia a gente se descontrai juntos”, - “.. o curso servirá para crescimento e melhor rendimento de todos”.

Nestes tópicos foram evidenciados o sentimento de valorização como pessoa, ligado ao auto-conhecimento e a necessidade de se trabalhar com as próprias emoções para a vida diária.

O relacionamento interpessoal foi abordado em termos de contacto com pacientes, colegas, superiores hierárquicos e outras pessoas da UNICAMP, sobressaindo a integração, aproximação e convivência.

Em relação à instituição/trabalho foi destacada a importância da união a setores e de hierarquias diferentes, bem como de se entender melhor o ambiente de trabalho com seus problemas para melhora do rendimento de todos.

\section{SAÚDE FÍSICA/MENTAL}

A busca pela saúde, através da diminuição do stress e portanto a valorização da pessoa humana como um todo, foi destacada como importante segundo as falas abaixo:

. “... bom para meus problemas de saúde”, - "... comecei a perceber e educar minha dinâmica corporal”, - “.. mostrou como é possível eliminar tensões do dia-a-dia, fazendo a auto-massagem e respiração completa", - "... foi uma forma de desligarmos um pouco do dia-a-dia stressado que temos", - “... amenizou e muito o nosso cansaço físico e mental", - "... deu para relaxar bem e esquecer os problemas", - "Estou me sentindo super bem com os exercicios".

Os depoimentos revelam a situação árdua do trabalho em hospital e a relevância de se proporcionar oportunidades para que as pessoas possam tomar consciência da importância da sua saúde e de evitar o stress. Isto foi captado por uma participante que disse: "Nunca pensei que depois de 30 anos de trabalho muito desgastante, pudesse encontrar algum momento que me ajudase tanto. Me ajudou a crescer espiritualmente. Hoje já estou pronta para trabalhar mais 30 anos se precisar". 


\section{APRENDIZAGEM}

Dentro da perspectiva da educação a oportunidade é uma condição fundamental para a ocorrência da aprendizagem, no sentido de conscientização para mudança de comportamento, e pode ser verificada pelas falas: “... aprendi muita coisa que não tinha idéia”, - “... a gente aprende a se cuidar, a dar mais valor ao próprio corpo...", - "Aprendi com os professores que para cuidar dos pacientes é preciso eu cuidar de mim antes e descobri vários pontos sensíveis em meu corpo", - "Aprendi coisas que não imaginava ver, ouvir e participar", - “.. Aprendi a me sentir bem à vontade”, - “... Trouxeme oportunidades fantásticas, esclarecimentos nos aspectos sobre o indivíduo, o meio e a espiritualidade e também a técnica de como lidar com os sentimentos", - "Funcionária que sou do HC há mais de 14 anos nunca me foi dada a oportunidade de participar de um curso deste nível, onde aprendi muito".

O resultado mostra que, tanto a oportunidade como a aprendizagem, em alguns aspectos, pode ser alcançada através desses cursos alternativos.

\section{APLICABILIDADE}

Considerando a necessidade da aplicação dos conhecimentos no cotidiano, os cursos apresentaram um caráter eminentemente prático, como afirmam: - “... tudo que aprendi estou praticando pelo menos 10 minutos por dia.", - “... estava passando por uma fase de grande stress, me adaptando ao novo trabalho e usei os exercicios em casa, o que ajudou a relaxar.”, - “.. praticando em casa me senti melhor durante o trabalho, na enfermaria me lembrava e aplicava, como na hora de mobilizar paciente no leito e em outras intercorrências". Assim parece reforçar o aspecto da aplicabilidade.

\section{REFLEXÃO}

Estas falas: - ".. fez com que refletíssemos em tudo que nos estressavam", - ".. Tenho refletido mais sobre os meus atos e de outros com quem convivo, sem precisar estar achando isto ou aquilo", - “.. fez uma alerta em minha vida, deixou-me mais sensível a algumas reflexões", - "A aula convivência humana me fez ver, meu interior, como está minha convivência em casa, no trabalho e no mundo", refletem um repensar sobre o individuo, o trabalho, a convivência e a vida.

\section{SOLUÇÃO DE PROBLEMAS}

Muitos problemas podem ser solucionados através de técnicas simples que visem atingir o indivíduo de forma integral, como relataram: - 'Estou respirando melhor, dormindo melhor, principalmente tinha muita insônia e cefaléia”, - “.. auxiliou muito a livrarmos do cansaço do dia-a-dia”, - “.. senti mais vida, mais força para vencer os obstáculos do dia-a-dia, pois há 
muitos anos eu estava apagada e este curso me acendeu novamente”, - “... estou desempenhando minhas funções com mais segurança na minha vida particular".

Portanto, solucionar problemas pode ser a busca do bem estar e melhoria de qualidade de vida das pessoas.

\section{PONTO NEGATIVO}

O único ponto levantado como negativo foi referente ao local: - "Não gostei do espaço onde foi realizado o curso, poderia ser em outro lugar". Esta observação é procedente, pois para execução das atividades foi necessária a retirada de todas as carteiras da sala, a cada aula, o que acarretava certos transtornos, já que algumas atividades, como por exemplo a dança, exigiam espaço mais amplo.

\section{SUGESTÕES}

As sugestões apresentadas estão descritas na TABELA 2.

TABELA 2. Sugestões dadas por participantes dos cursos alternativos. Ano 1992.

\begin{tabular}{|c|c|c|}
\hline SUGESTÕES & $\begin{array}{c}\mathrm{N}^{\circ} \\
(\mathrm{PESSOAS})\end{array}$ & $\%$ \\
\hline
\end{tabular}

Continuidade dos cursos

$\begin{array}{rr}36 & 45,0 \\ 18 & 22,5 \\ 9 & 11,3 \\ 5 & 6,2 \\ 6 & 7,5 \\ 4 & 5,0 \\ 2 & 2,5\end{array}$

Oportunidade para todos funcionários 18

Avaliação e Aprofundamento de Conteúdo 9

Aumentar a carga horária

Continuar dentro do horário de trabalho

Inserir "Relaxamento Mental" no dia a dia

Não deram sugestões

80

100,0

TOTAL DE DEPOENTES

45,0

11,3

6,2

7,5

5,0

2,5

Fonte: Cursos alternativos Depto. Enf. - FCM e SEEC - HC - UNICAMP.

Dentre as sugestões dadas pelos participantes dos cursos alternativos, a maior ênfase foi quanto à continuidade dos mesmos $(45,0 \%)$ e a oportunidade para todos os funcionários $(22,5 \%)$ perfazendo um total de mais de $67,0 \%$. 


\section{CONCLUSÃo}

Para concluir este estudo, a fala de uma das depoentes pode nos dar uma idéia do sentido que perpassou os cursos como um todo e da responsabilidade da instituição em promover, não apenas os "treinamentos tradicionais", mas atividades educativas do ponto de vista participativo que conduzam aos cuidados com a saúde do pessoal de Enfermagem, numa perspectiva holística, na qual a mente, a emoção, o intelecto e o corpo se relacionam como um todo e com tudo, a exemplo do que ocorreu neste trabalho.

- "Nós da enfermagem, que trabalhamos quase todo o tempo sob condições adversas, acrescentando-se a isso que a maioria somos mulheres que cumprem dupla jornada de trabalho (hospital e lar), ocasionando um esgotamento físico/mental muito grande, chegamos a esquecer de nós mesmas, como ser humano único, com anseios, tensões e necessidades próprias. Foi bom saber que conlamos com pessoas que se preocupam com este fato $e$ idealizaram um curso que nos coloca frente a frente com o problema e nos dá alternativas de como enfrentá-los..... até mesmo a descoberta de que somos capazes de expressar corporalmente, foram temas benéficos para o nosso corpo e espírito. Ajudou a compreender a causa de uma série de males físicos e como atenuá-los. Até o lazer ganhou novas perspectivas".

A avaliação feita pelos depoentes demonstrou que a "vivência" em grupo, facilitou o relacionamento entre os membros da equipe de trabalho, abriu espaço para comunicação efetiva; favoreceu o auto-conhecimento e ajudou na solução de problemas.

Além destes pontos ficou evidente a importância da continuidade de cursos alternativos extensivos a todas as pessoas do hospital, como uma das formas de auxiliar o funcionário na melhoria do relacionamento consigo mesmo, com o outro e com o trabalho e de valorizar a sua saúde física e mental e a aprendizagem.

\section{RECOMENDAÇÕES}

Considerando que este foi um trabalho preliminar sobre a experiência vivida pelos funcionários e estes autores, através dos cursos alternativos, recomenda-se que sejam implementadas as sugestões dadas pelos pesquisados e elaborem outros estudos com esta temática, dando continuidade e profundidade a esta e outras pesquisas. 
MADEIRA, C.G. et al. Health and Education: alternative courses for the development of nursing personnel. Rev.Esc.Enf.USP, v.30, n.2, p.217-28, aug. 1996.

To promote therapeutic educational activities for nursing personnel in order to decrease stress, to improve interpersonal relations and the search for self-knowledge are the objectives of the courses promoted by the Departament of Nursing of FCM and by The Continued Education Nursing Service of the University Hospital of UNICAMP. Respiration, relaxation, body sensibilization and awareness, and theater interpretation techniques were taught in the following courses: The Hospital and Human Relations, Dance and Creativity, Yoga and Mental Relaxation. The estrategy employed was "group experience", with the participation of nurse's aides, nurses technicians, practical nurses, and registered nurses during working hours in 15 to 20 meetings per course. "Individual statements" written by the participants were used as a research tool, and the method employed was content analysis. The evaluation demonstrated that the "experience" facilitated relations among the members of the work team, opened the space for effective communication, favored self-knowledge, and helped with the problem solving. The analysis demonstrated the importance of the continuity of alternative courses to help employes improve their relations with themselves, with others and with their work, and to properly value their health/ learning.

UNITERMS: Education nursing.Interpersonal relations.

Educations continuing.

\section{REFERÊNCIAS BIBLIOGRÁFICAS}

01. BARDIN, L. Análise de conteúdo. Lisboa, Edições 70, 1977.

02. CAPRA, F. O Ponto de mutação. São Paulo, Cultrix, 1982.

03. KOLLER, E.M.P. et al. Reflexão sobre a prática atual da enfermagem e prenúncios de mudanças para o século XXI. Rev.Bras.Enf., v.45, n. 1, p. 74-9, 1992.

04. LUDKE, M. et al. Pesquisa em educação: abordagens qualitativas. São Paulo, EPU, 1986.

05. MADEIRA, C.G. et al. Funções do enfermeiro (a) em unidades de cuidados intensivos e semi-intensivos: subsídios para reflexão e mudanças. In: CONGRESSO BRASILEIRO DE ENFERMAGEM, 44., Brasilia. 1992. Anais. Brasilia, ABEn-Seção DF, 1992. p.5.

06. MINAYO, M.C. de S. O desafio do conhecimento: pesquisa qualitativa em saúde. São Paulo-Rio de Janeiro, Hucitec/Abrasco, 1992.

07. NOGUEIRA, M.J. de C. Abordagem holística: uma proposta para a enfermagem brasileira In: CONGRESSO BRASILEIRO DE ENFERMAGEM, 37, Recife, 1985. Anais. Recife, ABEn-Seção PE, 1986. p.508-26.

08. SEVO, W.A. A Administração da nova era. Rev.Planeta, março, 1992.

09. SILVA, M.J.P. et al. Educaça continuada: estratégia para o desenvolvimento do pessoal de enfermagem. Rio de Janeiro. Marques-Saraiva, 1989.

10. TREVISAN, M.A. et al. Liderança do enfermeiro no contexto hospitalar: expectativas do pessoal. Rev.Bras.Enf, v.44, n.1, p.55-9, 1991. 